\title{
GESTIÓN DEL CONOCIMIENTO Y RIESGOS DE DESASTRES DESDE UNA PERSPECTIVA LOCAL
}

\author{
(Disasters Risk and Knowledge's Management, \\ from a Local Perspective)
}

Carmen Delia Almaguer Riverón, Allan Pierra Conde

* Facultad de Humanidades, Instituto Superior Minero Metalúrgico, Moa, Holguín, Cuba. Calmaguer@ismm.edu.cu

(Recibido Noviembre 20 de 2006 y aceptado Mayo 14 de 2007)

\begin{abstract}
Resumen:
Trata sobre la evaluación de los riesgos de desastres desde la perspectiva del desarrollo local en el municipio de Moa (Holguín, Cuba). Los estudios realizados forman parte de un proyecto de investigación que llevan a cabo especialistas del área de sociales de la Facultad de Humanidades y del Centro de Energía Minero Ambiental. Durante los estudios se ejecutaron consultas y encuestas a la comunidad y a los organismos de la región, las cuales aportaron resultados significativos que sirven de base para tomar medidas y contribuir al desarrollo minero energético de la región de Moa. Es un trabajo que puede tomarse como modelo en otras regiones del país.
\end{abstract}

Palabras clave: Riesgos, Desastres, Desarrollo minero.

\section{Abstract:}

It deals with the disasters risks' evaluation, from the Moa municipality local development's perspective, in Holguín, Cuba. The studies made are part of an investigation project carried out by specialists of the Social Area of the Humanities School and the Environmental Mining Energy's Center. Some consultations and surveys to the community and the regional organisms were done, which contributed with significant results to the studies, base upon which measures were taken to contribute to the mining development of the Moa region. It is a model that can be applied in other regions of the country.

\section{INTRODUCCIÓN}

A ctualmente se reconoce que los desastres están de una u otra forma relacionados con una suma de prácticas humanas inadecuadas, resultado de una mala planificación y ejecución del desarrollo, que provoca impactos diferenciados en la sociedad; desde este punto de vista, la relación desarrollo-desastre es estrecha, y un avance en la solución del problema pasa necesariamente por un proceso de gestión del conocimiento como condición básica para proyectar el desarrollo sostenible a nivel local.

Con la investigación se aspira a:

- Incrementar el actual desempeño del Centro de Gestión del Riesgo del territorio.

- Capacitar a todos los actores locales en una concepción amplia del desastre.

- Incrementar la cultura de prevención de desastres sobre la base de la modelación de las percepciones sociales de los peligros identificados para el territorio.

- Ampliar la capacidad de respuesta de los diversos sectores de la población, particularmente de aquellos en condiciones altas de vulnerabilidad, frente a los peligros naturales y tecnológicos.
- Construcción de los escenarios de riesgo en el territorio a partir del uso de la prospectiva estratégica.

- Diseño de una política eficaz de comunicación del riesgo para el territorio, que tenga en cuenta a todos sus ciudadanos e incorpore la perspectiva de género.

Una de las cuestiones más importantes por desarrollar en los marcos del proyecto es la gestión prospectiva del riesgo, porque requiere de herramientas metodológicas, así como de un alto grado de conciencia, preocupación y compromiso con la reducción del riesgo por parte de todos los actores sociales implicados en el desarrollo local sostenible.

\section{MATERIALES Y MÉTODOS}

Los cambios en el planeta se manifiestan a través de fenómenos naturales como los sismos, los huracanes, las intensas lluvias, las prolongadas sequías y otros, cuyas consecuencias se hacen sentir en mayor medida en los países subdesarrollados. Estos fenómenos se convierten en peligros debido al crecimiento acelerado de la población, a la ausencia de procesos adecuados de planificación de la urbanización y uso de los territorios y, en general, a la irracionalidad imperante en los modelos de desarrollo. En opinión de Cardona (2003). 
... La disminución del impacto de los desastres debe enfrentarse primordialmente bajo la acción concertada y decidida de mejorar o cambiar el estilo o modelo de desarrollo, modelo que debe continuar dirigiéndose a mejorar la calidad de vida de todos, pero al mismo tiempo, encontrando la manera de disminuir vulnerabilidades y de buscar el equilibrio entre las necesidades de progreso y la preservación del medio ambiente. Es necesaria una acción concertada, donde a los aspectos técnico-científicos se les valide como necesarios, pero no como suficientes. Es necesario enfatizar que existe una estrecha relación entre el mal manejo del medio ambiente y el incremento de desastres...

Un desastre es, en opinión de Lavell (2006),

el fin de un proceso, a veces muy largo, de construcción de condiciones de riesgo en la sociedad. El desastre es la realización o concreción de las condiciones de riesgo preexistentes en la sociedad. Esta realización ocurre en el momento en que un determinado evento físico, sea un huracán, sismo, explosión, incendio u otro, ocurre y con ello muestra las condiciones de vulnerabilidad existentes...

es entonces cuando puede comprenderse cómo el riesgo, latente hasta ese momento, se convierte en un producto con consecuencias que se traducen en pérdidas y daños que pueden llegar a ser cuantiosos en términos financieros y siempre impactantes desde el punto de vista sociopsicológico.

Con referencia a la problemática particular de los desastres, el riesgo se refiere a un contexto caracterizado por la probabilidad de pérdidas y daños que abarcan tanto las particularidades físicas como psicosociales y culturales. El riesgo constituye, según Lavell, una probabilidad de daños relacionados con la existencia de determinadas condiciones en la sociedad, y que abarca a individuos, familias, comunidades, ciudades, infraestructura productiva y vivienda, entre otros, por lo que resulta oportuno destacar cómo el factor humano, sus percepciones y representaciones sociales sobre el riesgo ante situaciones de desastres adquieren gran importancia para modelar e instrumentar estrategias de comunicación del riesgo en contexto que tributen a la preparación individual y social, cuestión que requiere tanto del conocimiento estructurado como del no estructurado.

Por lo anterior, se entiende que el tema de los riesgos y los desastres está estrechamente ligado con problemas del desarrollo, es decir, con desequilibrios entre el ambiente social, el ambiente construido y el ambiente natural de un territorio, con lo que aumenta la susceptibilidad y la exposición a diferentes tipos de peligros. De igual forma, emerge una relación directa entre condiciones de vulnerabilidad y procesos inadecuados de apropiación del conocimiento o reconocimiento del propio entorno que requieren de nuevos conceptos y prácticas educativas.

Las Naciones Unidas, al instituir el Decenio Internacional para la Reducción de los Desastres Naturales (1990-1999), consideró la necesidad de trabajar por modificar las concepciones hasta ahora imperantes sobre los desastres, así como por incluir la gestión del riesgo en la perspectiva del desarrollo sostenible a nivel territorial.

El riesgo global, total o de desastre se manifiesta en territorios definidos y circunscritos, y es sufrido por individuos, familias, colectividades humanas, sistemas productivos o infraestructuras ubicados en sitios determinados. Los desastres tienen una expresión territorial definida que varía entre lo muy local hasta cubrir vastas extensiones de un país o varios países, por lo que, atendiendo a los criterios de Lavell, podrían considerarse un aspecto esencial en la definición de estrategias de desarrollo local sostenible.

En tal sentido, el enfoque de la Gestión de Riesgo en los territorios representa un proceso social complejo a través del cual se pretende lograr la reducción de los niveles de riesgo existentes en la sociedad y fomentar procesos de construcción de nuevas oportunidades de producción y asentamiento en el territorio en condiciones de seguridad y sostenibilidad aceptables.

En el año 2005, en Japón, la Conferencia Mundial sobre la Reducción de los Desastres aprobó el Marco de Acción de Hyogo para el decenio 2005-2015: Aumento de la resiliencia de las naciones y las comunidades ante los desastres. En él se plantea:

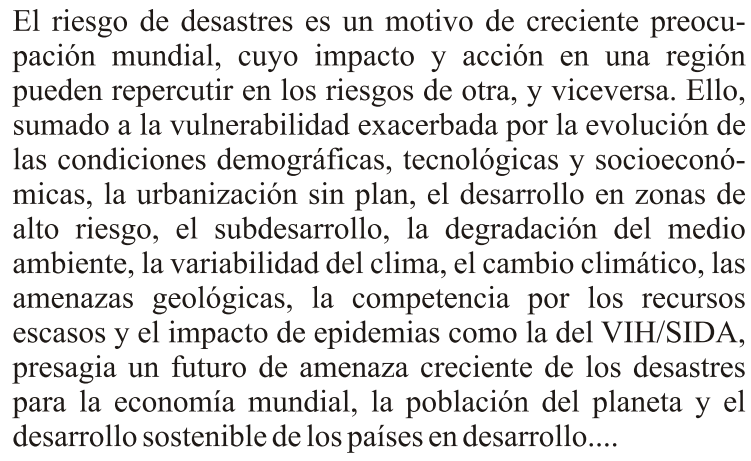

En el referido documento, las acciones que se consideraron pertinentes para el decenio 2005-2015 fueron:

- Gobernanza: marcos institucionales, jurídicos y normativos. - Identificación, evaluación y vigilancia de los riesgos y alerta temprana.

- Gestión de los conocimientos y educación.

- Reducción de los factores de riesgo subyacentes.

- Preparación para una respuesta eficaz y una recuperación efectiva.

El acceso al conocimiento, su difusión y aplicación consecuente en contextos no solo resultan necesarios para alcanzar el desarrollo, sino también para alcanzar el control sobre los procesos tecnológicos y la regulación del riesgo. Ante la "invisibilidad" de los riesgos, es el saber lo que permite "reconocerlos" y "darles existencia". Sin embargo, el saber también puede negarlos o transformarlos, ya sea minimizándolos o dramatizándolos, como afirma Gutiérrez en su artículo "América Latina ante la Sociedad del Riesgo". 
Es por eso que el conocimiento es hoy un factor necesario para el desarrollo, pero también es un requisito básico para intervenir en el control de la distribución de los riesgos, en un mundo en el cual la difusión de los peligros generados en buena medida por el desarrollo "industrial" es supranacional y frecuentemente desconoce las soberanías nacionales, como plantea el propio cambio climático.

\section{RESULTADOS}

\subsection{Experiencia de la gestión del riesgo en Cuba}

La Defensa Civil en Cuba centra la problemática de los desastres y constituye un sistema de medidas defensivas de carácter estatal programado para enfrentar situaciones de emergencia en el país, con el propósito de proteger a la población y la economía contra la destrucción causada por los desastres naturales y antrópicos.

A pesar de las limitaciones económicas imperantes en el país y a la frecuencia e intensidad de fenómenos de carácter hidrometeorológico, el país cuenta con fortalezas para el desarrollo de la gestión del riesgo. Entre estas fortalezas se consideran, según Rodríguez y Pérez (2004), las siguientes:

- La voluntad política que prioriza la temática.

- Las sinergias alcanzadas entre instituciones científicas, de ordenamiento territorial, de la vivienda, de defensa civil y universidades.

- La identificación de políticas, estrategias y medidas para la prevención, mitigación, preparación y respuesta a diversos plazos temporales que incluyen la rehabilitación y reconstrucción ante los desastres.

- La existencia de leyes, decretos leyes de defensa civil (ley 75/1994 de la Defensa Civil y decreto ley 170/1997 del Sistema de Medidas de Defensa Civil) y normas constructivas.

- La planificación del desarrollo y existencia de un proceso inversionista conciliado con la Defensa Civil.

- El fortalecimiento de las capacidades institucionales y humanas.

- La existencia de un sistemas de predicción, monitoreo y vigilancia de las amenazas.

- La formulación de planes de reducción de desastres.

- La organización de la población.

- La información y capitación de la población alcanzados.

Frente a estas potencialidades, los autores antes citados señalan que existen insuficiencias debidamente identificadas, resultantes del desarrollo histórico de los asentamientos poblacionales y de factores económicos, que se convierten en fuertes amenazas para el logro de la gestión del riesgo en determinados territorios del país.

Pudieran considerarse, además, otras insuficiencias, entre ellas, incluirse la carencia de estudios sobre las percepciones sobre el riesgo y de la memoria histórica de sus pobladores sobre estos, así como en términos generales de la cultura sobre riesgos en el nivel local; esta idea es desarrollada ampliamente por Omar D. Cardona en su artículo “¿Cultura de la prevención de desastres?" (2003), al plantear
... no hay aún una teoría que pueda hacer afirmaciones concluyentes acerca de cómo la población, en forma individual o colectiva, tiene una lectura del riesgo. Se puede afirmar que en general los 'imaginarios' varían notablemente de un sitio a otro o de una comunidad a otra.

Sin embargo, excepto en el caso de personas fatalistas, que leen adversidad incluso en aspectos que no la reflejan, en general se puede decir que existe una aversión instintiva al riesgo, que se traduce en una subestimación o negación implícita de las personas a verse involucradas en situaciones de peligro. Tal como se mencionó previamente, el riesgo se percibe para los demás y en muchas ocasiones, curiosamente, se rechaza o se minimiza sin fundamento hacia sí mismo; particularmente en relación con las amenazas de la naturaleza.

Estas ideas sugieren la necesidad del estudio de percepciones sociales del riesgo en los territorios, así como de la gestión del conocimiento para disminuir los niveles de vulnerabilidad existentes y garantizar en mayor medida la seguridad y calidad de vida de población.

\subsection{Desarrollo local sostenible y gestión del conocimiento para el "Centro de Gestión del Riesgo" en el municipio de Moa}

El logro de los Objetivos de Desarrollo del Milenio (ODM) requiere articular procesos de gestión del conocimiento que permitan la gestión eficaz del riesgo en función del desarrollo en sus diferentes escalas y contextos.

En el espacio local, los riesgos difícilmente pueden separarse, están ligados a los procesos agrícolas, al manejo de suelos, de construcción de viviendas, desarrollo del turismo y otros, son parte integrante del desarrollo local; cuestión sensible en los territorios que desarrollan actividades mineras, por las características e importancia que reviste esta actividad económica.

En el desarrollo local de comunidades mineras, la minería genera impactos tanto positivos como negativos en el modo de vida de la comunidad, en los flujos humanos y financieros, así como en el medio ambiente, generando e incrementando los peligros y vulnerabilidades de sus habitantes.

Se evidencia, de este modo, una transacción que se debe identificar, evaluar y considerar al momento de enfrentar el tipo de desarrollo local que se dará en un territorio particular, como lo es el municipio de Moa.

El municipio de Moa se encuentra, según la regionalización económica de Cuba, realizada por Propín (1992), en la Macrorregión Económica Oriental, formando parte de la subunidad taxonómica regional Guantánamo-Moa-Baracoa (Mesorregión), que posee características socioeconómicas mixtas agroindustriales y está compuesta por territorios predominantemente montañosos, donde su base industrial encuentra sus expresiones más acentuadas en la agroindustria especializada en el cultivo del café y la rama azucarera. Se distingue el caso del municipio de Moa por poseer una estructura económica polarizada en la minería no ferrosa, reportando también actividad en la rama química. 
La extracción minera es un ejemplo de producción que presenta muy diversos riesgos, pero que se desarrolla en función de alcanzar altísimos beneficios en tiempos cortos; en tal sentido, el desarrollo minero metalúrgico de este municipio y su ubicación geográfica hacen del territorio uno de los más expuestos en Cuba a una serie de peligros tanto naturales como tecnológicos, de ahí la creación de un Centro de Gestión del Riesgo en Moa.

El fortalecimiento de la capacidad de Cuba para el desarrollo de la gestión del riesgo incluye en su primera etapa la creación de Centros de Gestión de Riesgo en los 50 municipios de mayor exposición a los peligros naturales o tecnológicos del país; actualmente están en funcionamiento 17 Centros de Gestión de Riesgo. En las provincias orientales, debido a sus particularidades, se encuentran ubicados dos de estos Centros, uno en el municipio de Moa, perteneciente a la provincia Holguín, y otro en Guamá, en la provincia de Santiago de Cuba.

Los Centros de Gestión de Riesgo (CGR) son un espacio físico con un equipamiento modular designado para facilitar a las autoridades del territorio el manejo de los riesgos de desastres, influyendo directamente en la reducción de las vulnerabilidades y en el fomento de una cultura de prevención de desastres y paralelamente en la preparación de la sociedad para enfrentar los peligros que puedan afectar a la población, los recursos económicos y la infraestructura del territorio.

Los CGR tienen entre sus funciones la creación de:

- Bases de datos sobre la vulnerabilidad de los diferentes sectores del territorio (vivienda, industria, agropecuario, educación, salud, comunicaciones, cultura, viales y otros).

- Datos georreferenciados con el empleo de un SIG (Sistema de Información Geográfica), que permitan analizar sistemáticamente las vulnerabilidades del territorio y la adopción de medidas para la reducción de desastres.

- Datos históricos sobre las diferentes situaciones de desastres ocurridas en el territorio.

- Estudios de peligro y vulnerabilidad, así como evaluaciones de riesgo que se hayan realizado en entidades del territorio.

Para dar respuesta de manera eficiente a sus funciones, los CGR necesitan instrumentar procesos de gestión del conocimiento que permitan abordar en toda su complejidad la problemática del riesgo, y propicien la implementación, seguimiento y evaluación de políticas y programas sociales orientados a la reducción de la vulnerabilidad y la sostenibilidad de los territorios.

Deberá tenerse en cuenta que el riesgo se puede representar de múltiples formas, de acuerdo con su escala y su dimensión social, económica, ambiental o política. En consecuencia, se deben redoblar los esfuerzos para desarrollar indicadores apropiados para expresar las múltiples facetas del riesgo en todas las escalas.

Se requiere, además, mejorar la forma de traducir entre los distintos actores sociales las múltiples dimensiones del riesgo, generar un lenguaje común y educar a las generaciones futuras acerca del riesgo y la manera de reducirlo, teniendo en cuenta que las soluciones macro, a nivel internacional, no son suficientes para lograr la reducción del riesgo a nivel local.

La gestión del conocimiento a nivel local deberá comprenderse como un proceso complejo de generación, asimilación, administración y circulación de informaciones, datos, saberes y valores necesarios que garanticen en su aplicación la solución de los problemas de carácter local y contribuyan así a la elevación de la calidad de vida de la población sobre la base del desarrollo sostenible y la participación ciudadana.

La gestión del conocimiento en el desempeño del Centro de Gestión del Riesgo en Moa deberá permitir:

- Proporcionar información clara sobre los riesgos de desastre y las distintas formas de protección, en correspondencia con las percepciones sociales de sus pobladores, incorporando la perspectiva de género y los factores culturales y sociales que caracterizan a este territorio minero.

- Fortalecer las redes entre los expertos, los planificadores y los encargados de la gestión en materia de desastres, reforzando los procedimientos para utilizar los conocimientos especializados y tradicionales disponibles e incorporando en mayor medida a profesionales de las ciencias sociales y humanísticas.

- Fomentar el diálogo y la cooperación entre el Gobierno Local, las entidades productivas y de servicios, la Sede Central (Instituto Superior Minero Metalúrgico), la Sede Universitaria del territorio, centros de investigación, de proyectos, de capacitación $\mathrm{y}$, en sentido general, a todos los profesionales que desde diferentes perspectivas teóricas y metodológicas se ocupan de la reducción de los riesgos de desastre, alentando la conformación de una red de conocimientos para la temática.

- Promover el uso, la aplicación y la asequibilidad de las últimas tecnologías de la información y la comunicación y las tecnologías espaciales y los servicios conexos, así como las observaciones terrestres, para contribuir a la reducción del riesgo de desastre, en particular para la formación y para el intercambio y la divulgación de información entre las distintas categorías de usuarios.

Por los aspectos anteriormente mencionados -en el ámbito de la educación formal y no formal-, se vislumbran amplios derroteros no solo para hacer análisis de riesgos en disciplinas y contextos específicos, aspecto que constituye un elemento vital para abordar el territorio y sus dinámicas sociales en un contexto más amplio de hábitat, sino también para avanzar en el conocimiento técnico y científico de manera que se den las premisas para generar investigaciones adecuadas para la región y la generación de un capital humano más comprometido con la reducción de la vulnerabilidad física, cultural y social.

El Programa Ramal del Ministerio de Educación Superior en Cuba "Gestión Universitaria del Conocimiento y la Innovación para el Desarrollo" (GUCID) constituye el marco idóneo para la conformación de redes de conocimiento para la solución de los problemas inherentes al desarrollo local sostenible de los 
territorios, incluyendo como línea de trabajo los estudios de peligro, vulnerabilidad y riesgo e incorporando en ellos a la Sede Universitaria, en su carácter de actor del desarrollo local.

La Sede Universitaria aglutina buena parte de los profesionales del territorio y deberá contribuir sustancialmente a ampliar la capacidad técnica y social para responder, desde las diferentes carreras y disciplinas, y en particular desde las ciencias sociales y humanísticas, a las necesidades del contexto y de los diversos sectores de la población, fundamentalmente de aquellos que viven en condiciones altas de vulnerabilidad por su exposición y susceptibilidad ante peligros de carácter natural y tecnológico.

La "nueva universidad cubana" amplía su encargo social al propiciar soluciones a partir de la gestión del conocimiento en función de los "Estudios de Peligro, Vulnerabilidad y Riesgo", así como en el desarrollo de los denominados institucionalmente "Centros de Gestión del Riesgo" (CGR).

En tal sentido, la existencia de la Sede Universitaria de Moa, como universidad en el territorio que forma profesionales en carreras de Licenciatura en Psicología, Sociología, Comunicación Social, Derecho, Estudios Socioculturales y Contabilidad, junto al Instituto Superior Minero Metalúrgico, constituye una fortaleza para el desempeño del Centro de Gestión del Riesgo en el Municipio una vez que se tiene en cuenta el carácter cada vez más cognoscitivo de todas las actividades económicas, técnicas, sociales y políticas, así como la rapidez de los cambios en estos campos y que hacen que la información sea la materia prima a procesar e integrar en conocimiento.

Promover la gestión eficiente del conocimiento comprende el establecimiento de un acceso equilibrado a los conocimientos relevantes para incrementar la calidad de vida de los ciudadanos a nivel de los territorios y se alcanza no solo mediante la formación profesional, sino desarrollando también capacidades para producir, difundir y aplicar conocimientos y extenderlos al resto de los procesos sustantivos inherentes a la universidad en el denominado modelo contexto-céntrico, concepto introducido por Souza et ál. (2001).

La Sede Universitaria Municipal de Moa debe desempeñar, entonces, un rol protagónico en el desarrollo sostenible del territorio, y al igual que los demás agentes de la comunidad está obligada a pensar, a reformular conceptos, a promover la investigación participativa y la investigación-acción, a explorar nuevos caminos, asumiendo la dinámica del desarrollo socioeconómico en el contexto minero de Moa.

La posibilidad de contar con la ayuda del conocimiento acumulado a partir del nivel local hasta el regional, sobre la evaluación del riesgo y la vulnerabilidad, forma la base de la construcción de políticas de desarrollo que incorporan la probabilidad de ocurrencia de desastres. Cuando a escala local existen datos e información puntual, es más fácil prevenir desastres, así es que las bases de datos y las evaluaciones del riesgo poseen un valor adicional, y su ausencia hace imposible conocer la geografía cambiante del riesgo y los factores que forman o producen la vulnerabilidad y el daño.

Los instrumentos de medición que se han desarrollado permiten combinar información extraída de diversos contextos para armar este rompecabezas de un desarrollo humano sostenible que incorpore el riesgo y la vulnerabilidad a nivel local.

Al asumir las consideraciones expuestas y ante la necesidad de contribuir desde la gestión del conocimiento al desarrollo del Centro de Gestión del Riesgo en Moa, el Proyecto "Estrategias de desarrollo local sostenible para los municipios del Este de la Provincia Holguín a partir de la gestión del conocimiento por las SUM" incorpora la problemática del riesgo ante situaciones de desastres naturales y tecnológicos como una línea temática por desarrollar con la participación del Instituto Superior Minero Metalúrgico de Moa (Sede Central) y su centro de Estudios de Medio Ambiente.

\section{CONCLUSIONES}

- La gestión del conocimiento para el desarrollo local sostenible deberá incorporar el tema de los riesgos y los desastres al estar estos estrechamente ligados con problemas históricos del desarrollo, con desequilibrios entre el ambiente social, el ambiente construido y el ambiente natural.

- El acceso al conocimiento, su difusión y aplicación consecuente en contextos, no solo resultan necesarios para alcanzar el desarrollo, sino también para alcanzar el control sobre los procesos tecnológicos y la regulación del riesgo frente a probables desastres naturales o tecnológicos.

- El Programa Ramal del Ministerio de Educación Superior en Cuba, "Gestión Universitaria del Conocimiento y la Innovación para el Desarrollo" (GUCID), constituye una excelente oportunidad para la conformación de redes de conocimiento para la solución de las problemáticas inherentes al desarrollo local sostenible de los territorios, incluyendo los estudios de peligro, vulnerabilidad y riesgo al incorporar a la Sede Universitaria como actor del desarrollo local.

- La Sede Universitaria aglutina buena parte de los profesionales del territorio y deberá contribuir sustancialmente a ampliar la capacidad técnica y social para responder, desde las diferentes carreras y disciplinas, y en particular desde las ciencias sociales y humanísticas, a las necesidades del contexto y de los diversos sectores de la población, fundamentalmente de aquellos que viven en condiciones altas de vulnerabilidad por su exposición y susceptibilidad ante peligros de carácter natural y tecnológico.

- El proyecto en desarrollo, "Gestión del conocimiento para el Centro de Gestión del Riesgo en el municipio Moa”, constituye una línea de trabajo en función del desarrollo local sostenible de este territorio minero. 


\section{AGRADECIMIENTOS}

Los autores desean expresar un agradecimiento a todos los colaboradores en esta investigación, y a los analistas de la Facultad de Humanidades del ISMM.

\section{REFERENCIAS}

Cardona A., Omar Darío (2003). ¿Cultura de la prevención de desastres? En: Seguridad Sostenible. Gobernanza y Seguridad Sostenible. Una colección del IIGC. Edición 10, martes 22 de abril de 2003. Consultado 20/7/2006 en: http://www.iigov.org/ss/article.drt?edi=181749\&art= 181756

Coca, Claudia P. Iniciativas de Educación Superior en Gestión de Riesgos en Bogotá. Consultado 4/5/2006 en: http:// www.ucv.ve/comir/documentos/habitat\%20y\%20riesgo/ ASPECTOS $\% 20$ ACADEMICOS/PONENCIAS/36\%20 $\% 20 I N I C I A T I V A S \% 20 \mathrm{DE} \% 20$ EDUCACION\%20SUPE RIOR\%20EN\%20BOGOTA.\%20Claudia\%20Coca\%20 Colombia.doc

EMNDC (2006). Aspectos a tener en cuenta para la creación y el funcionamiento de los Centros de Gestión para la Reducción de los Riesgos.

Gutiérrez, Ileana. América Latina ante la Sociedad del Riesgo. Consultado 16/7/2006 en: http://www.oei.es/salactsi/ gutierrez.htm

Lage, A. (2005). Intervención en el Taller Nacional sobre Gestión del Conocimiento en la Nueva Universidad.

Lavell, A. (2006). Sobre la Gestión del Riesgo: Apuntes hacía una definición. Consultado: 12/6/2006 en: http:// www.crid.or.cr/crid/CD_Inversion/pdf/spa/doc15036/doc 15036-contenido.pdf
Lavell, A, y Arguello R., Manuel (2003). Gestión de riesgo: un enfoque prospectivo. Tegucigalpa: PNUD, Consultado 18/7/2006 en: http://www.crid.or.cr/crid/CD_Inversion/ $\mathrm{pdf} / \mathrm{spa} / \mathrm{doc} 14681 / \mathrm{doc} 14681$-indice.pdf

Marco de Acción de Hyogo (2005-2015). Aumento de la resiliencia de las naciones y las comunidades ante los desastres. Consultado: 12/6/2006 en: http://www.unisdr.org/ eng/hfa/docs/Hyogo-framework-for-action-spanish.pdf

Núñez, J. J. (2003). Indicadores y relevancia social del conocimiento. Ponencia en el Primer Taller de Indicadores de Percepción Pública, Cultura Científica y Participación Ciudadana. Salamanca, Consultado 21/7/2006 en: http://www.ricyt.org/interior/normalizacion\%5Cpercepci on publica/6.pdf

Núñez Jover, J., Félix, M. y Pérez H., L. (2006). La gestión del conocimiento, la ciencia, la tecnología y la innovación en la nueva universidad: una aproximación conceptual. En: La nueva universidad cubana y su contribución a la universalización del conocimiento. La Habana: Editorial Félix Varela. pp. 5-20.

Organización de Naciones Unidas. Decenio internacional para la reducción de los desastres naturales: nuevas disposiciones. Informe del Secretario General Organización de Naciones Unidas. Objetivos del Milenio. Consultado 16/ 7/2006 en http://www.un.org/spanish/millenniumgoals/

Propín, E. (1992). Problemas metódicos de la regionalización económica de Cuba. Tesis presentada en opción al grado científico de Doctor en Ciencias Geográficas, La Habana.

Rodríguez, C. M, Pérez H, Luisa. (2004). Componentes de la gestión del riesgo en la prevención de desastres naturales. Caso Cuba. Jornadas Iberoamericanas sobre hábitat, vulnerabilidad y desastres. Centro de Formación de la Cooperación Española en Santa Cruz de La Sierra, Bolivia Consultado: 16/7/2006, en: http://www.yorku.ca/hdrnet/ images/uploaded/RODRIGUEZ_04\%5B1\%5D.pdf 